\title{
Acrochordonous plaque in a Doberman Pinscher dog
}

\author{
Ariele A. Ferreira ${ }^{1, *}$, Rodrigo Friesen ${ }^{2}$, Juliana K. Oliveira ${ }^{1}$, William Timboni Teixeira', Thiago Sillas ${ }^{2}$, \\ Juliana Werner ${ }^{3}$, Jorge Luiz Costa Castro ${ }^{4}$ and Peterson T. Dornbusch ${ }^{1}$ \\ ${ }^{1}$ Department of Veterinary Medicine, Federal University of Paraná, Rua dos Funcionários, Curitiba, Brazil \\ ${ }^{2}$ Clinivet Hospital Veterinário, Rua Holanda, Curitiba, Brazil \\ ${ }^{3}$ Werner \& Werner-Laboratório de Patologia Animal, Rua Tomazina, Curitiba, Brazil \\ ${ }^{4}$ Pontifícia Universidade Católica do Paraná, Rua Rockfeller, Curitiba, Brazil
}

\begin{abstract}
An 8-yr old, neutered female Doberman Pinscher was presented for dermatological evaluation due to numerous pruritic, non-pigmented nodules that created a plaque in the lumbo-sacral region. This report is the first published photographic record of an acrochordonous plaque in a dolichocephalic dog and is the first reported case in Brazil.
\end{abstract}

Keywords: Acrochordons, Benign skin tumors, Fibroepithelial polyps.

\section{Introduction}

Acrochordons or fiproepithelial polyps are benign skin tumors, with a clinical presentation that varies from single to multiple nodules, exophytic to pedunculated and lesions can occur in dermal and epidermal tissue (Bidaut et al., 2003). These benign tumors are rare in dogs, however, they are common in human beings. Dermal and epidermal hyperplasia can occur as single or multiple lesions, however, when multiple small acrochordons coalesce an acrochordonous plaque is formed (Bidaut et al., 2003; Gross et al., 2005).

Acrochordonous plaques are composed of a coalescence of filiform polyps forming a painless and non-pruritic mass (Gross et al., 2005). The etiology of this disease still uncertain in dogs although chronic traumatic lesions or healed furunculosis can be predisposing factors (Bidaut et al., 2003; Gross et al., 2005). Acrochordons can affect dogs of all ages but are more frequently diagnosed in dogs more than 5-yr old. In the published reports, males are more frequently reported than females. Lesions have been reported more frequently on limbs than other body sites (Goldschmidt and Goldschmidt, 2017).

Acrochordons are well described in human dermatology and can be accompanied by other skin disorders, such as fibrofolliculomas and trichodiscomas, being a part of Birt-Hogg-Dubé syndrome (Birt et al., 1977). Acrochordons were originally described as a part of this syndrome, however, they may occur in isolation (Lee et al., 2005).

Acrochordonous plaques are characterized by numerous small acrochordons, with mature collagen and usually vascularization. The superjacent epidermis shows acanthosis, hyperkeratosis, and hyperpigmentation and usually with formation of rete ridges. Furthermore, it is common to see dermal inflammatory infiltrates, the absence of hair follicles and adnexal glands and the lesion may be surrounded by dermal fibrosis. These benign tumors have a unique morphological appearance, making histopathological diagnosis of acrochordon and acrochordonous plaque straightforward (Gross et al., 2005). This is the first Brazilian case of an acrochordonous plaque in a female Doberman. The case presents an unusual clinical presentation and is illustrated with the first photographic record of this skin disorder.

\section{Case Details}

An 8-yr old, neutered female Doberman Pinscher was presented with a skin disorder comprising numerous pruritic non-pigmented coalescent nodules arising from the epidermis. The lesion formed a plaque in the lumbo-sacral region that had progressed over 60 d. The clinical appearance is shown in Figure 1. No fleas were seen nor reported by the owner. Considering the clinical appearance and presentation, the main differential diagnosis were acrochordonous plaque, nodular dermatofibrosis, and squamous cell carcinoma. A fine needle aspirate was performed and cytological examination of a May-Grünwald-Giemsa stained sample demonstrated high cellularity, mostly mature typical anucleated keratinocytes with keratinous debris between cell clusters, and suggestive of an epifollicular cyst.

Treatment with $22 \mathrm{mg} / \mathrm{kg}$ cefalexin twice daily (Rilexine $^{\circledR}$, Virbac, Carros, France) and $0.75 \mathrm{mg} /$ $\mathrm{kg}$ prednisone once daily (Meticorten ${ }^{\circledR}$, Merck Sharp \& Dohme Farmacêutica Ltda, São Paulo, Brazil) was prescribed. During $15 \mathrm{~d}$ of follow-up, the lesion increased in size, measuring a diameter of $15 \mathrm{~cm}$, and surgical excision of the entire plaque was performed. Following surgical excision, there was resolution 


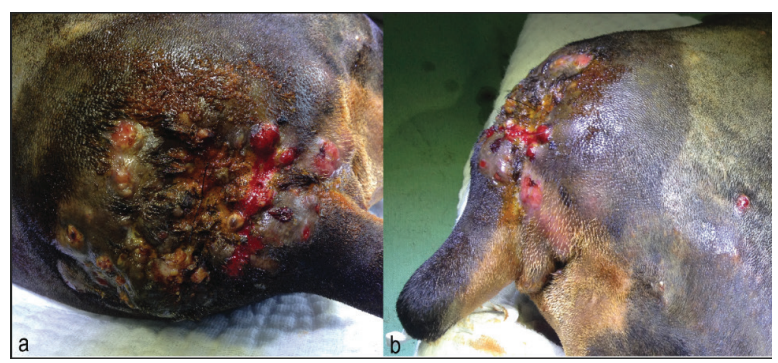

Fig. 1. An 8-yr old, neutered female, Doberman Pinscher with multiple, non-pigmented and coalescent nodules, forming a $20 \mathrm{~cm}$ diameter plaque. (a) Clinical appearance of acrochordonous plaque, note areas of skin erosion and purulent discharge, induced by pruritus and self-trauma. (b) Lateral view of acrochordonous plaque, showing skin lesion thickness.

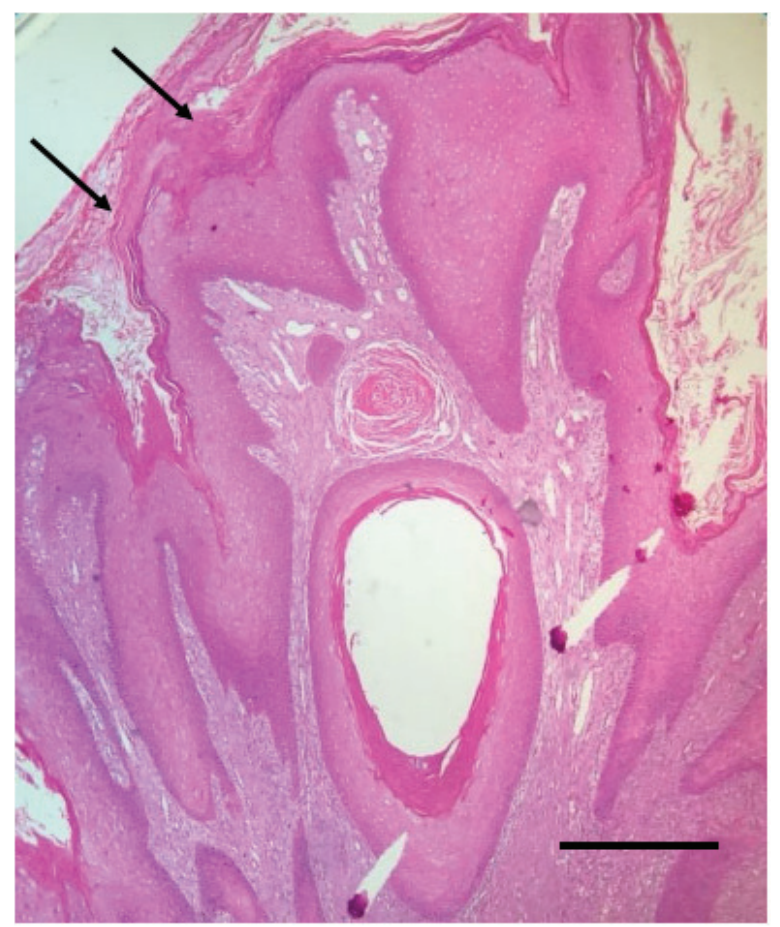

Fig. 2. Histopathological photomicrograph of acrochordonous plaque in a dog stained with hematoxylin and eosin (40× objective, scale bar, $60 \mu \mathrm{m}$ ). Note the adjacent filiform exophytic sessile projections surrounded by hyperplastic epidermis with compact laminar orthokeratosis (black arrows). Within the dermis, there is collagen proliferation and absence of adnexal glands.

of the clinical signs and no recurrence after 6 mo of follow-up. The excised skin sample was fixed in $10 \%$ formalin, paraffin embedded, cut in $5 \mu \mathrm{m}$ sections and stained with hematoxylin and eosin for histopathologic evaluation.

Histopathologic features of the skin lesion comprised adjacent exophytic filiform sessile projections, surrounding by hyperplastic epidermis with laminar orthokeratosis and intense basket weave hyperkeratosis. In the adjacent dermis, there was an edematous collagenous tissue proliferation which was well differentiated with a perpendicular orientation to collagen fibers in the epidermis a diffuse perivascular pattern of mixed inflammatory infiltrate was also present. Where hair follicles were present there was infundibular hyperkeratosis. These features are compatible with the diagnosis of fibroepithelial polyp plaque, also known as acrochordonous plaque, in this case the histological features were also compatible with chronic trauma, possibly as a result of the pruritic nature of the lesion. The histopathologic presentation is shown in Figure 2, in which the rete ridges, characteristic of this skin lesion, can be seen.

\section{Discussion}

Acrochordons rarely affect dogs, and there is only one case series where they are reported to form the acrochordonous plaque (Bidaut et al., 2003), which first suggested the term for this skin condition. That case series comprised three male dogs, with ages ranging from 3 to $6 \mathrm{yr}$, suggesting a predisposition for young male dogs. However, the small number of cases reported, makes it hard to draw conclusions on gender, age, and breed predispositions. It was originally hypothesized that brachycephalic breeds were predisposed (Bidaut et al., 2003) since the first reports were in two English Bulldogs and one Pug. However, another report refers to a Pit bull Terrier (Gross et al., 2005). In our case, the breed affected was a dolichocephalic female dog, in disagreement with the breed and gender predisposition suggested, and being 8-yr old, was older than previous reports.

Single acrochordons usually occur on limbs (Goldschmidt and Goldschmidt, 2017); however, previously acrochordonous plaques have been described on the head, lateral, and dorsal cervical regions and tail base (Bidaut et al., 2003). Our case occurred in the lumbo-sacral region, which is similar to the tail base location reported in the Pug. Although the region affected in this case is similar to that reported in the Pug, in our case, this was an area without skin folds, in contrast to the Pug. This case demonstrates that the lesion may occur in a multitude of locations. The lumbo-sacral area is known to be the most affected region for flea allergic dermatitis (Bruet et al., 2012), and although the owners and the veterinarian did not report fleas, it is not possible to completely discard flea allergic dermatitis as a predisposing factor for pruritus and self-trauma, and consequent acrochordonous plaque development.

Acrochordonous plaques have clinical signs that vary from non-pruritic to low-grade pruritus and are painless (Bidaut et al., 2003; Gross et al., 2005); however, in our case, the dog had intense pruritus and pain around the skin disorder. The pruritus developed after the plaque formation and was probably due to secondary 
inflammation and infection. The main differential for clinical presentation of multiple fibropruritic nodules is nodular dermatofibrosis, which can ulcerate and be pruriginous due to secondary folliculitis and/or furunculosis (Gross et al., 2005). Besides that, the clinical appearance also suggested squamous cell carcinoma, which can have similar presentation such as plaque-like formation, large diameter, multiple nodules, alopecia, and ulceration (Gross et al., 2005). Considering singular nodules, such as polypoid fibromas, polypoid hemangiomas, granulation tissuetype hemagiomas, lymphangiomas, also can be similar to acrochordons. However, acrochordonous plaque has a unique morphological appearance on histopathology, which differentiate from other fibropruritic nodules and gives the definitive diagnosis (Gross et al., 2005). Acrochordonous plaques can also be related to traumatic lesions and deep skin infection (furunculosis) (Bidaut et al., 2003; Gross et al., 2005). In our case, histopathologic findings suggested a lesion, probably secondary to the intense pruritus.

\section{Conclusion}

Acrochordonous plaque is a rare skin condition in dogs and this case report describes some important differences with respect to previous reports, i.e., a novel area of skin affected and variation from previously reported breed and gender predisposition. Complete surgical excision was curative for this case.

\section{Acknowledgments}

The authors would like to thank Clinivet Veterinary Hospital and Werner \& Werner Pathology Laboratory.

\section{Conflict of interest}

The authors declare that they have no conflicts of interest.

\section{References}

Bidaut, A.P., Gross, T.L., Noli, C., Welle, M. and Suter, M.M. 2003. Case report: Acrochordonous plaques in two Bulldogs and a Pug dog. Vet. Dermatol. 14, 177-179.

Birt, A.R., Hogg, G.R. and Dubé, J. 1977. Hereditary multiple fibrofolliculoma with trichodiscomas and acrochordons. Arch. Dermatol. 113, 1674-1677.

Bruet, V., Bourdeau, P.J., Roussel, A., Imparato, L. and Desfontis, J.C. 2012. Characterization of pruritus in canine atopic dermatitis, flea bite hypersensitivity and flea infestation and its role in diagnosis. Vet. Dermatol. 23, 487-e93.

Goldschmidt, M.H. and Goldschmidt, K.H. 2017. Epithelial and melanocytic tumors of the skin. Ed., Meuten DM. Tumors in domestic animals. 5th Edn., Hoboken, NJ: Wiley-Blackwell, pp: 88-141.

Gross, T.L., Ihrke, P.J., Walder, E.J. and Affolter, V.K. 2005. Fibrous tumors. Eds., Gross TL, Ihrke PJ. Skin diseases of the dog and cat: clinical and histopathological diagnosis. 2nd Edn., Oxford, UK: Blackwell Science, pp: 710-734.

Lee, D.A., Grossman, M.E., Schneiderman, P. and Celebi, J.T. 2005. Genetics of skin appendage neoplasms and related syndromes. J. Med. Genet. 42, 811-819. 